\title{
Tumor Burden and Intraosseous Metabolic Activity as Predictors of Bone Marrow Failure during Radioisotope Therapy in Metastasized Prostate Cancer Patients
}

\author{
Francesco Fiz, ${ }^{1,2}$ Samine Sahbai, ${ }^{3}$ Cristina Campi, ${ }^{4}$ \\ Matthias Weissinger, ${ }^{1}$ Helmut Dittmann, ${ }^{1}$ Cecilia Marini, ${ }^{5}$ Michele Piana, ${ }^{6}$ \\ Gianmario Sambuceti, ${ }^{7}$ and Christian la Fougère ${ }^{1}$ \\ ${ }^{1}$ Nuclear Medicine Unit, Department of Radiology, University of Tübingen, Tübingen, Germany \\ ${ }^{2}$ Department of Internal Medicine, University of Genoa, Genova, Italy \\ ${ }^{3}$ Nuclear Medicine Unit, "Henri Mondor" University Hospital, Paris, France \\ ${ }^{4}$ National Council of Research-SPIN, Section of Genoa, Genova, Italy \\ ${ }^{5}$ National Council of Research-IBFM, Section of Genoa, Genova, Italy \\ ${ }^{6}$ Department of Mathematics, University of Genoa, Genova, Italy \\ ${ }^{7}$ Nuclear Medicine Unit, Department of Health Sciences, University of Genoa, Genova, Italy \\ Correspondence should be addressed to Francesco Fiz; francesco.fiz.nm@gmail.com
}

Received 7 August 2017; Accepted 6 December 2017; Published 25 December 2017

Academic Editor: Kazuma Ogawa

Copyright (C) 2017 Francesco Fiz et al. This is an open access article distributed under the Creative Commons Attribution License, which permits unrestricted use, distribution, and reproduction in any medium, provided the original work is properly cited.

\begin{abstract}
Rationale. Radium-223-Dichloride (Ra-223) is an alpha-emitter, used to treat bone metastases. Patients with high metastatic burden and/or with increased trabecular bone uptake could present a higher incidence of hematologic toxicity. We hypothesized that these two factors are predictors of bone marrow failure. Material and Methods. A computer algorithm discriminated between trabecular bone $\left(B_{\mathrm{Vol}}\right)$ and tumor metastases $\left(M_{\mathrm{Vol}}\right)$ within pretherapeutic whole-body skeletal SPECT/CT $(N=47)$. The program calculated the metastatic invasion percent (INV\%) as the $M_{\mathrm{Vol}} /\left(M_{\mathrm{Vol}}+B_{\mathrm{Vol}}\right)$ ratio and extracted the $B_{\mathrm{Vol}}$ mean counts. $B_{\mathrm{Vol}}$ counts were correlated to \% drop of hemoglobin (Hb), leukocytes (WBC), and platelets (PLT) after 3/6 Ra-223 cycles. Patient-specific and computational-derived parameters were tested as predictors of hematologic toxicity with MANOVA. Results. $B_{\mathrm{Vol}}$ counts correlated with drop of $\mathrm{Hb}(R=0,65, p<0.01)$ and PLT $(R=0,45, p<0.01)$. Appendicular $B_{\mathrm{Vol}}$ counts showed a better correlation $(p<0.05$, $p<0.01$, and $p<0.001$ for Hb, WBC, and PLT, resp.). INV\% directly correlated with $B_{\mathrm{Vol}}$ counts $(R=0.68, p<0.001)$. At MANOVA, grade III/IV toxicity was predicted by INV\% $(p<0.01)$, by long-bone invasion $(p<0.005)$, and by $B_{\mathrm{Vol}}$ counts $(p<0.05)$. Conclusions. In patients with significant bone tumor burden, degree of bone invasion and trabecular bone uptake are predictors of subsequent bone marrow failure.
\end{abstract}

\section{Introduction}

Patients affected by castration-resistant prostate cancer (CRPC) present an extremelyhigh incidence of skeletal metastases [1]. In recent years, numerous novel treatment modalities have been developed to treat metastasized CRPC [25]. Among these, radionuclide therapy with alpha-emitting Ra-223-Dichloride (Ra-223) has been recently approved [6].
This isotope allows obtaining a significant survival improvement, alongside with satisfying pain palliation and reduction of skeletal-related events [7-11]. Moreover, Ra-223 therapy, owing to its short emission radius [12], is characterized by a relatively low incidence of hematologic toxicity, when compared with $\beta$-emitting radioisotopes [13, 14]. In fact, Ra223-related bone marrow failure presents an incidence of 3$4 \%$ for grade III and $1 \%$ for grade IV reactions [10]. 
However, these data are derived from a heterogeneous CRPC population, whose patients presented very diverse tumor burden, ranging from 2 to $>20$ metastases. Actually, more than half of the enrolled population had less than 20 skeletal localizations [10]. Recent studies have however suggested that the incidence of bone marrow failure could be more common in patients presenting a higher number of skeletal metastases $[6,15,16]$.

Two main factors could influence the onset of bone marrow toxicity during the Ra-223 therapy: the tumor burden per se and the metabolic intensity of nontarget trabecular bone.

In fact, under normal conditions, active bone marrow takes up about one-third of the available volume within the intrabone space; this "red" bone marrow is located within the vertebrae, the sternum, and the flat bones [17]. If this space is invaded by metastases, hematopoietic stem cells can home and seed in more distal hematopoietic niches in the appendicular skeleton. As the disease inevitably progresses, bone metastases are increasingly observed in the appendicular skeleton as well. In this scenario, the space for healthy bone marrow becomes also restricted and necessary compensatory bone marrow expansion (e.g., because of Ra-223 induced damage) is hampered.

On the other hand, radiotracer uptake within the trabecular skeleton, where the hematopoietic bone marrow is located, can present significant interpatient variations $[18,19]$. As Ra-223 uptake tends to mirror the one of the common bone-seeking tracers $[8,20]$, a higher metabolism in the pretherapeutic bone scintigraphy could entail a greater $\mathrm{Ra}$ 223 uptake as well as a longer residence time within the trabecular bone, possibly resulting in an overall higher dose to the bone marrow.

The first point, that is, correlation between tumor burden and bone marrow failure, was tested by studies employing an automated thresholding in bone scans [21] or in PET [22]. Though interesting, the PET-threshold approach is scannerspecific, requiring center-specific validation as well as periodic recalibrations, and is therefore not readily exportable; the planar analysis is on the other hand subject to summation artifacts [23].

With regard to the second point, the correlation between bone tracer uptake intensity and hematologic toxicity remains to this day substantially unexplored.

In this paper, we applied a novel computational tool to hybrid SPECT/CT imaging, to measure the overall tumor volume and the radioactivity concentration in the bone marrow. This method uses a segmentation algorithm to identify the trabecular bone and the metastases in the CT images, thus avoiding the problems related to PET/scintigraphy thresholding. Then it can extract additional data from the coregistered metabolic images, in order to estimate the tracer concentration in these two volumes. The goal of the present investigation is to test the hypothesis that an enhanced intrabone mineral metabolism and an increased tumor burden can predict a higher risk for bone marrow toxicity. Moreover, the specific role of the bone marrow located within the appendicular skeleton, which might be key in patients with extensive axial bones metastasization, is also explored.

\section{Materials and Methods}

2.1. Patients' Population and Procedures. Forty-seven adult patients (mean age $69.5 \pm 7$, age range 55.5-80.8), consecutively admitted to our unit for pretherapeutic evaluation of skeletal metastases from CRPC were retrospectively analyzed. Inclusion criteria comprised histologically confirmed prostate neoplasia, evidence of prostate specific antigen (PSA) increase in course of maximal androgen blockade, and presence of clinically symptomatic and radiological confirmed skeletal metastases. Exclusion criteria were confirmed or suspected visceral metastases, presence of grade 2 hematologic toxicity at baseline, according to CTCAE criteria, version 4.0 [24], end-stage renal disease, imminent danger of pathologic fracture or unwillingness/incapacity to sign an informed consent. Any previous therapy or combination of treatments was admitted. Similarly, any entity of metastatic burden was conceded and no selection on the basis of number of metabolically active areas was performed.

Each patient underwent a baseline Tc-99m-dicarboxypropane-diphosphonate (DPD) whole-body bone SPECT/ CT scan, followed by six Ra-223-Dichloride injections (Xofigo ${ }^{\circledR}$, Bayer Pharma AG, Berlin, Germany, 50 KBq per Kg of body weight), each spaced one month apart. Hemoglobin, leukocytes (WBC), and platelets (PLT) were monitored on a weekly basis. Percent variation of these parameters was calculated after three and six Ra-223-Dichloride cycles.

All patients gave written informed consent for the retrospective analysis of the pseudonymized clinical SPECT/CT data. The investigations were conducted in accordance with the Helsinki Declaration and with national regulations, after approval by the ethics committee of the University of Tübingen.

2.2. Scan Protocol. Patients were scanned on a hybrid SPECT/CT device (Discovery 670 Pro, GE Healthcare, Chicago, US), three hours after injection of $8-10 \mathrm{MBq} / \mathrm{Kg}$ of Tc-99M-DPD (CIS Bio, Berlin, Germany). To minimize artifacts caused by the presence of radioactive urine in the excretory system, patients were asked to drink at least $1000 \mathrm{ml}$ of water during the uptake time and to void immediately before the scan.

The acquisition comprised a whole-body planar scan, followed by a whole-body SPECT/CT, from vertex up to the distal femoral epiphyses, obtained by reconstructing and fusing three sequential fields of view (Xeleris 3, GE Healthcare, Chicago, USA). SPECT acquisition was carried out with the two camera heads in H-Mode; parameters for each field of view were as follows: energy window $140.5 \pm 10 \%$, angular step $6^{\circ}$, time per step $15^{\prime \prime}$. The transaxial field of view and pixel size of the reconstructed SPECT images were $54 \mathrm{~cm}$ and $5 \times 5 \mathrm{~mm}$, respectively, with a matrix size of $128 \times 128$. SPECT raw data were reconstructed using OSEM iterative protocol ( 2 iterations, 10 subsets).

The 16-detector row, helical CT scanner used a gantry rotation speed of $0.8 \mathrm{~s}$ and a table speed of $20 \mathrm{~mm}$ per rotation, with a $120 \mathrm{kV}$ voltage and $10-80 \mathrm{~mA}$ current. A dose modulation system (OptiDose, GE Healthcare, Chicago, 


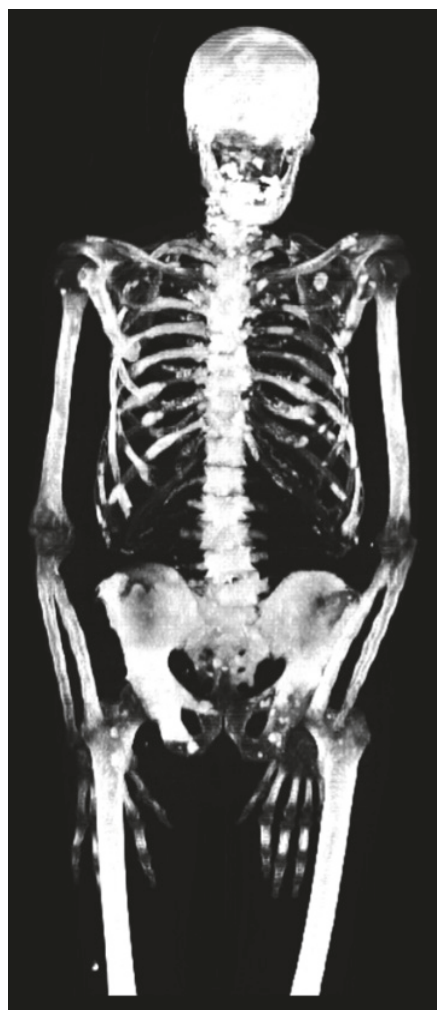

Whole-body SPECT/CT

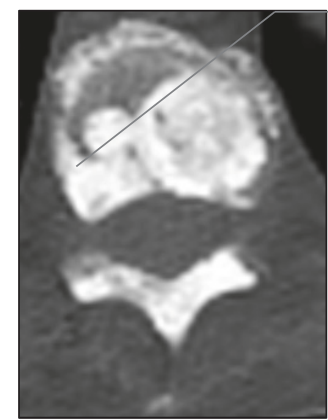

Original CT slice

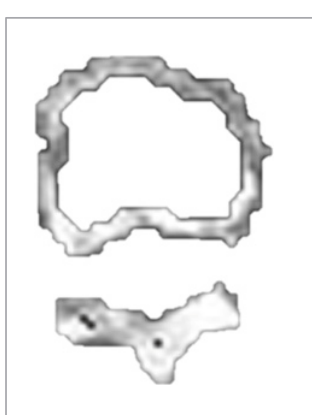

Segmented edge

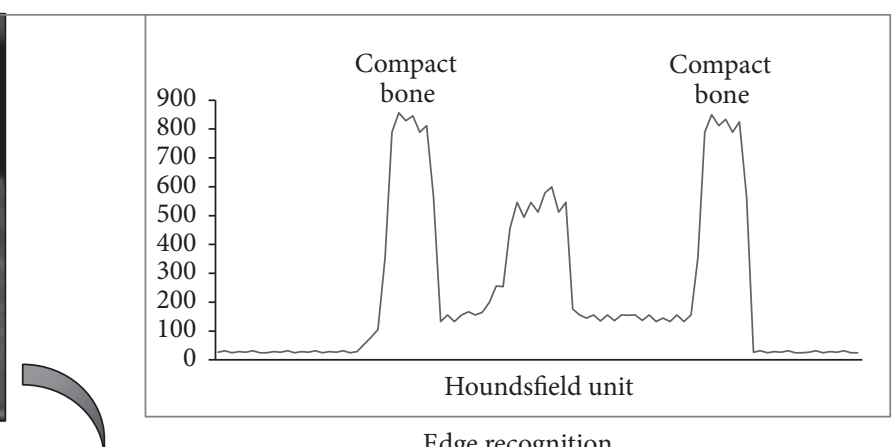

Edge recognition

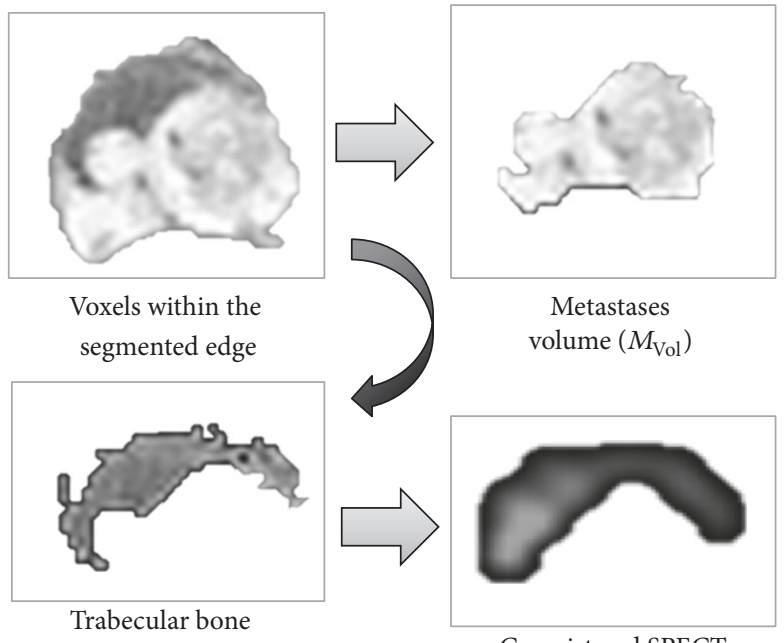

$\left(B_{\mathrm{Vol}}\right)$

Coregistered SPECT

FIGURE 1: Segmentation analysis of SPECT/CT images. The program detects the compact bone border, on a slice-by-slice basis, by identifying the abrupt change of Hounsfield value (segmented edge). The mean density value of this volume is used to tell apart the trabecular bone $\left(B_{\mathrm{Vol}}\right)$ from the metastases volume $\left(M_{\mathrm{Vol}}\right)$ on the voxels lying inside the detected surface. The program then generates two masks, corresponding to $B_{\mathrm{Vol}}$ and to $T_{\mathrm{Vol}}$, which are exported to the coregistered SPECT images, from which the functional information is extracted.

US) was applied to minimize total exposure according to the patient's size. No contrast medium was injected.

\subsection{Image Analysis. Segmentation of bone volumes on the} CT images was based on the previously validated method $[17,25]$. The computational software analysis tool was progressively developed and adapted by the joint effort of the Department of Mathematics and the Department of Health Sciences of the University of Genoa [17, 25-27]; it was later improved thanks to the collaboration with the Nuclear Medicine Unit of the University of Tübingen. The algorithm is based on the principle of segmentation analysis with adaptive threshold: it segments the osseous tissue not by aprioristically defining a Hounsfield value for bone, but by rather identifying the bone border, on each separate slice, by recognizing the sharp variation of attenuation value between soft and bone tissue.

Briefly, the algorithm identifies the skeleton on CT images by recognizing the sharp attenuation contrast between soft tissues and outer cortical bone. In normal subjects, two main components are identified: the outer compact bone and the intraosseous cancellous tissue, which can be recognized by the program in virtue of the attenuation shift between cortical and trabecular bone (Figure 1). In patients with bone metastases, a third volume can be identified, corresponding to the bone metastases. This volume can also be segmented, using the sharp contrast with the normal trabecular bone.

The bone recognition algorithm functions in two steps: in the first step the program identifies the outer margins of compact bone; from this point, it samples a two-pixel ring of skeletal tissue. Thereafter, all skeletal voxels lying internally to the compact bone volume and having attenuation coefficient equal or above the average compact bone density are considered as tumor metastases, while the remaining ones are labeled as normal trabecular bone (Figure 1). At the end of the first step, the program separates the three bone subcomponents, the compact bone, the trabecular bone $\left(B_{\mathrm{Vol}}\right)$, and the bone metastases $\left(M_{\mathrm{Vol}}\right)$, and calculates their volumes. Then, it proceeds to calculate the entity of metastatic invasion (INV\%), as the $M_{\mathrm{Vol}} /\left(M_{\mathrm{Vol}}+B_{\mathrm{Vol}}\right)$ ratio.

In the second step, the algorithm creates two masks, corresponding to $B_{\mathrm{Vol}}$ and to $M_{\mathrm{Vol}}$ and exports them to the coregistered SPECT images. Then, it measures the radioactivity concentration, expressed as mean counts, within these two volumes. 
The whole analysis is conducted first on the whole-body skeleton. Then, separate analyses are run on the bones of the axial skeleton (vertebrae and sternum) and for the long bones in the appendicular skeleton (humeral and femoral shafts). The costae are not analyzed, so as to prevent artifacts derived from the respiratory motion.

In order to detect patients presenting massive appendicular invasion (AI), as a sign of advanced disease, patients were stratified in two groups on the basis of the median value of appendicular INV\%: those in the upper half were considered to be AI-positive patients.

2.4. Statistical Analysis. The $t$-test for unpaired data was used to compare values between patients' subgroups. Correlation between indexes was assessed with bivariate analysis, using Pearson's $R$ index.

Differences in the events' occurrence between groups were tested using $X^{2}$ or Fisher's exact test, as appropriate. Multivariate analysis of variance (MANOVA) was used to test the influence of $B_{\mathrm{Vol}}$ and $M_{\mathrm{Vol}}$ counts, INV\%, body weight (as it reflects the administered activity), age, PSA values, "superscan," and AI on the occurrence of grade III/IV hematologic toxicity. A $p$ value of $<0.05$ was considered significant. The SPSS statistical program (SPSS ${ }^{\circledR}$, v. 21.0, IBM, Armonk NY, USA) was employed.

\section{Results}

3.1. Patients' Characteristics. 32 out of 47 patients presented high-risk disease [28] at diagnosis (68\%). Mean disease duration was $7.5 \pm 5.8$ years. On average, patients had known skeletal metastases since $36 \pm 26$ months; twelve patients (26\%) had a history of skeletal-related events. Mean time since hormone resistance onset was $30 \pm 23$ months and mean PSA at the time of the bone scan was $788 \pm 1843 \mathrm{ng} / \mathrm{ml}$. None of the patients was therapy-naive at the time of therapy; 34 $(72 \%)$ had a history of previous docetaxel therapy and 15 of them (32\%) had a history of skeletal radiation therapy. All patients were on antihormone therapy at the time of Ra223-therapy. All patients exhibited extensive metastasization: mean number of localizations, as observable on SPECTMIP, was $76 \pm 32$ (range 34-135). Patients' characteristics are detailed in Table 1.

3.2. Degree of Trabecular Bone Invasion and Uptake Distribution. Average INV\% was $28 \pm 20 \%$ (range $4-71 \%$ ). This figure did not exhibit significant variation between the axial $(36 \pm 28 \%$, range $4-78 \%)$ and the appendicular sites $(31 \pm 21 \%$, range $1-69 \%)$.

Mean counts were higher in $M_{\mathrm{Vol}}$ than in $B_{\mathrm{Vol}}$ and tended to be markedly higher in the axial than in the appendicular skeleton (Figure 2). 28 patients (59\%) had a "superscan" appearance (completely of nearly absent visualization of renal parenchyma in the planar bone scan).

A direct correlation was noted between INV\% and $B_{\mathrm{Vol}}$ counts $(R=0.68, p<0.001)$; this correlation was also observable when considering the axial and the appendicular skeleton separately $(R=0.65$ and $p<0.001 ; R=0.59$ and $p<0.01$, resp.) as shown in Figure 3.
TABle 1: Patients' characteristics.

\begin{tabular}{lcc}
\hline Feature & Mean & Range \\
\hline Age (years) & $69,5 \pm 7$ & $55-81$ \\
Weight (Kg) & $72,8 \pm 27,6$ & $44-111$ \\
PSA (ng/ml) & $788 \pm 1843$ & $3-9375$ \\
Gleason score & $8 \pm 1$ & $5-9$ \\
Disease duration (months) & $90,5 \pm 69$ & $13,7-275,8$ \\
Skeletal metastases duration & $36,3 \pm 26$ & $1,1-139,8$ \\
(months) & $30 \pm 23$ & $1,9-105$ \\
CRPC duration (months) & $34(72 \%)$ & - \\
Previous docetaxel & $12(26 \%)$ & - \\
Previous skeletal RT & $76 \pm 32$ & $34-135$ \\
Focal lesions at SPECT MIP & $12 \pm 2$ & $8,8-16,2$ \\
Mean baseline hemoglobin & $5876 \pm 1743$ & $3100-10940$ \\
Mean baseline leucocytes & $220 \pm 66$ & $71-351$ \\
Mean baseline platelets & &
\end{tabular}

3.3. Therapy Adherence and High-Grade Bone Marrow Toxicity. All patients underwent at least one Ra-223-injection, 45 (96\%) underwent the first three cycles, and 32 (75\%) completed the entire six cycles of therapy. Reasons for Ra223 discontinuation included skeletal progression at interim analysis by means of bone scan and CT $(n=5)$, spread to visceral organs $(n=4)$, occurrence of grade III or higher hematologic toxicity not amenable to control $(n=$ $4)$, serious infection requiring hospitalization $(n=1)$, or withdrawal of consent after therapy initiation $(n=1)$. For these reasons, hematologic data after three cycles are available for all patients, while blood counts after the complete sixcycle therapy are present for 32 patients (75\%) only.

After three cycles, four patients had grade III $\mathrm{Hb}$ toxicity; two of them discontinued the treatment while the other two continued under supportive therapy: after six cycles, one of them recovered while the other still met the grade III $\mathrm{Hb}$ toxicity criteria. Three further patients had developed grade III $\mathrm{Hb}$ toxicity after six cycles.

Grade III leukopenia/neutropenia was observed after three cycles in one patient, who subsequently recovered. One more patient developed a grade IV leukopenia/neutropenia after three Ra-223 injections.

In total, nine patients (19\%) showed an at least grade III bone marrow dysfunction at some point in the course of Ra223 treatment.

No differences in the occurrence of toxicity or in the entity of blood parameter drop were observed when categorizing the patients on the basis of previous docetaxel/radiotherapy.

3.4. Therapy-Associated Changes in Blood Counts. After three Ra-223 cycles, at least one parameter among Hb, WBC, and PLT had dropped by $10-50 \%$ in 39 patients (87\%); a drop of at least $50 \%$ in any parameter was instead observed in 14 patients (30\%). Correlation between whole-body $B_{\mathrm{Vol}}$ counts and drop of the above-cited parameter was direct (Hb: $R=$ 0.33 and $p<0.05$; WBC: $R=0.42$ and $p<0.01$ and PLT: $R=0.49$ and $p<0.001)$. At site-based analysis, 

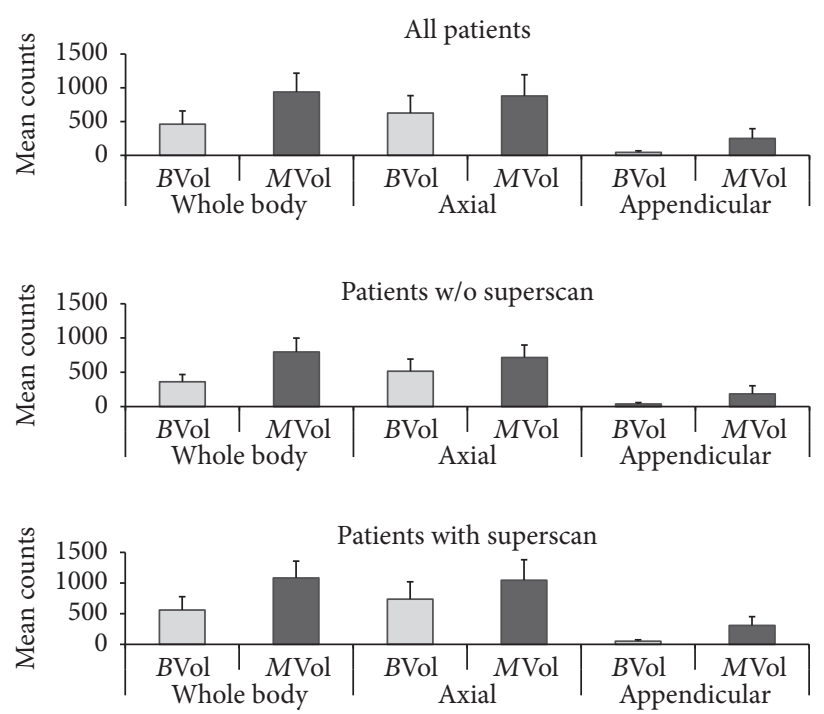

FIgURE 2: Mean counts in $B_{\mathrm{Vol}} / M_{\mathrm{Vol}}$ in whole-body, axial, and appendicular skeleton.
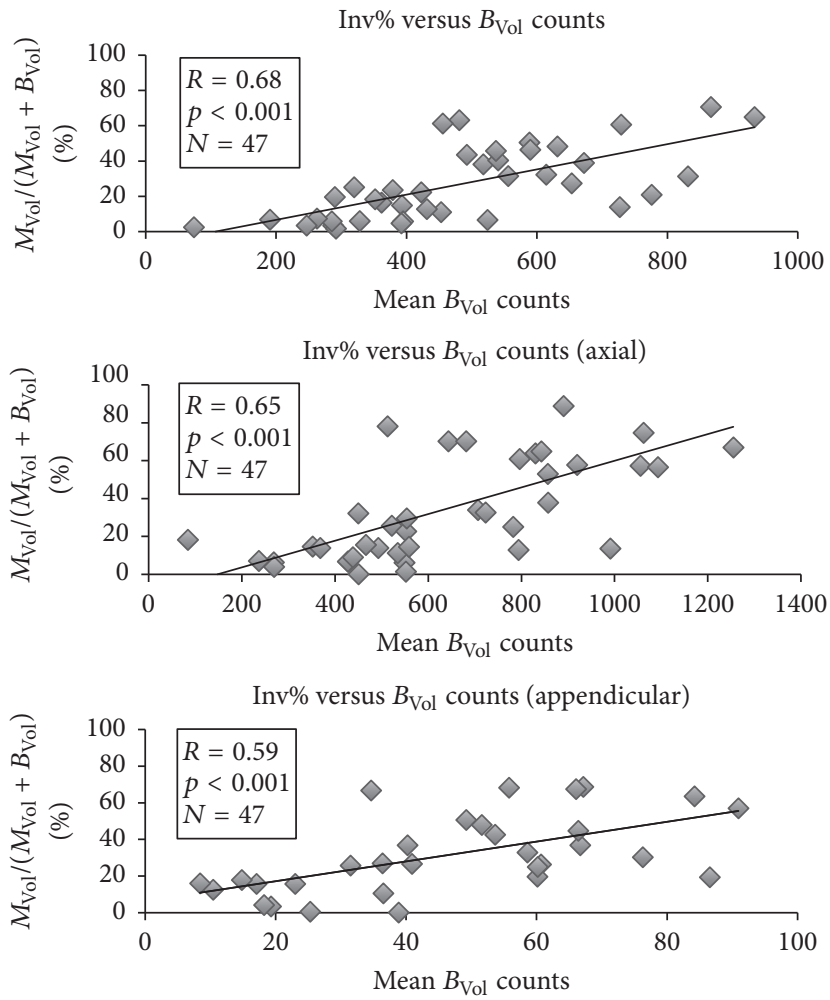

FIGURE 3: Correlation between percent metastatic invasion and mean uptake of unaffected trabecular bone. The entity of metastatic invasion positively and tightly correlates with bone uptake within trabecular bone. Said uptake can present significant variations among patients.

percent drop correlated with appendicular $B_{\mathrm{Vol}}$ mean counts (Hb: $p<0.05$; WBC: $p<0.001$ and PLT: $p<0.01$, Figure 4$)$. Conversely, correlation with axial $B_{\mathrm{Vol}}$ mean counts was looser and limited to WBC $(p<0.05)$.

At the end of the six cycles, $26 / 32$ patients (81\%) presented at least one parameter inferior by $10-50 \%$ with respect to baseline; $11 / 32(34 \%)$ showed a $\geq 50 \%$ drop. Correlation between whole-body $B_{\mathrm{Vol}}$ mean counts was particularly evident when considering variation of Hb and PLT $(R=$ 0.65 and $p=0.001$ for $\mathrm{Hb} ; R=0.45$ and $p<0.05$ for PLT). Similar to the interim evaluation, axial $B_{\mathrm{Vol}}$ mean counts did not significantly correlate with blood element variation; conversely, appendicular mean counts showed a robust association with the end-of-therapy $\mathrm{Hb}$, WBC, and PLT $(p<0.05,<0.01$, and $<0.001$, resp., Figure 4$)$. See Table 2 for the complete layout. 


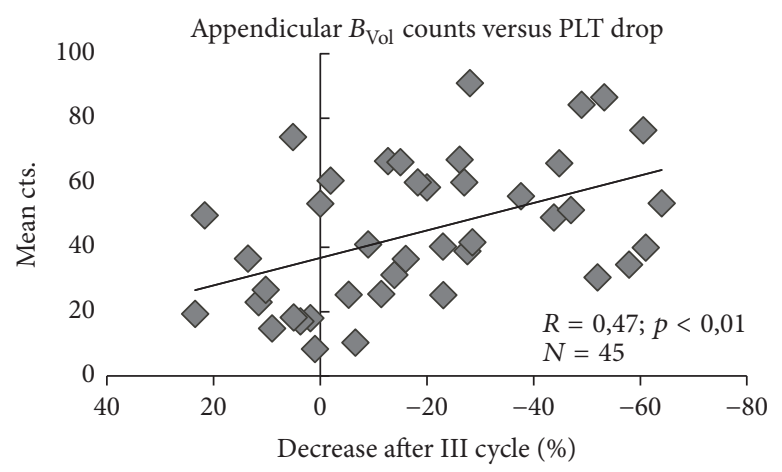

(a)

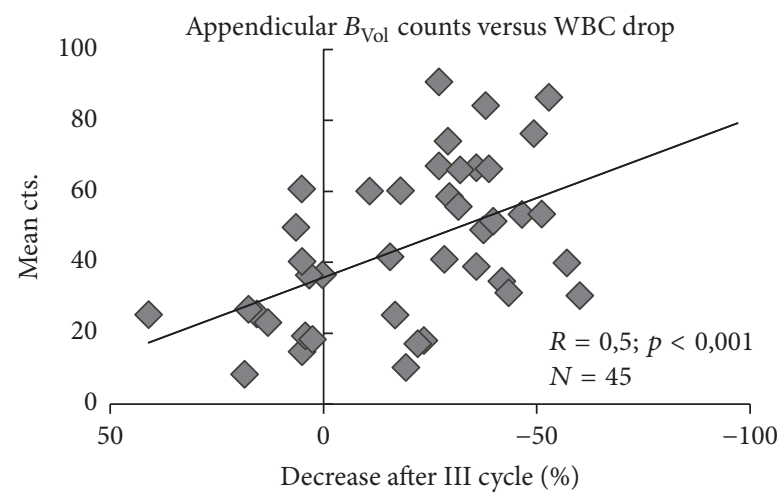

(c)

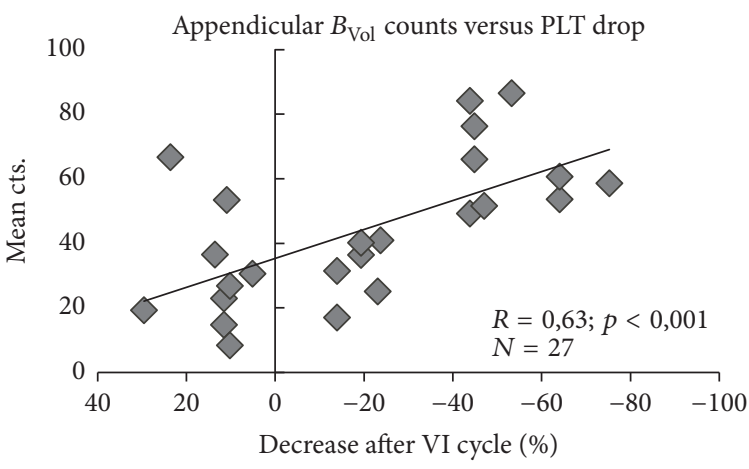

(b)

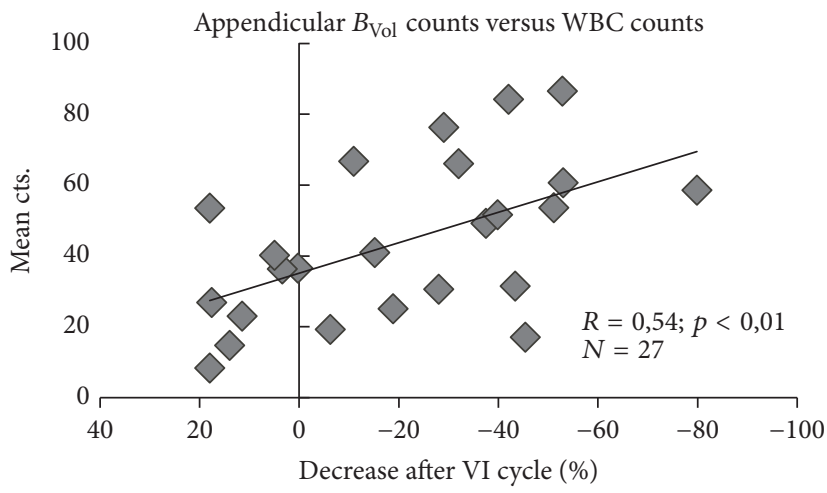

(d)

Figure 4: Correlation between appendicular $B_{\mathrm{Vol}}$ mean counts and blood values drop, after three (a, c) and six (b, d) cycles of Ra-223Dichloride. PLT = platelets; WBC = leukocytes.

TABLE 2: Correlation between trabecular mean counts and parameters drop.

\begin{tabular}{lccc}
\hline & $B_{\mathrm{Vol}}$ counts & AXIAL $B_{\mathrm{Vol}}$ counts & Appendicular $B_{\mathrm{Vol}}$ counts \\
\hline Hemoglobin (after 3rd cycle) & $-0,332^{*}$ & 0,023 & $-0,356^{*}$ \\
Leucocytes (after 3rd cycle) & $-0,423^{* *}$ & $-0,318^{*}$ & $-0,497^{* * *}$ \\
Platelets (after 3rd cycle) & $-0,479^{* * *}$ & $-0,295$ & $-0,473^{* *}$ \\
Hemoglobin (after 6th cycle) & $-0,644^{* *}$ & $-0,264$ & $-0,412^{*}$ \\
Leucocytes (after 6th cycle) & $-0,308$ & $-0,108$ & $-0,539^{* *}$ \\
Platelets (after 6th cycle) & $-0,451^{*}$ & $-0,254$ & $-0,628^{* * *}$ \\
\hline
\end{tabular}

${ }^{*} p<0.05 ;{ }^{* *} p<0.01 ;{ }^{* * *} p<0.001 ;$ MCs: mean counts.

With the exception of a sporadic association between appendicular mean counts and interim WBC $(p<0.05)$, values of $M_{\mathrm{Vol}}$ counts in any body region were not significantly correlated with hematologic variations (data not shown).

3.5. Role of Appendicular Invasion (AI). A significant AI was present in $23(49 \%)$ patients, as defined by the presence of an appendicular INV\% higher than the median value (i.e., $>27 \%$ ). Patients with AI had a more pronounced drop of all three parameters considered ( $\mathrm{Hb}, p<0.05$; WBC, $p<$ 0.001 ; and PLT, $p<0.01$ ) with respect to the remaining ones. Moreover, all the above-mentioned blood parameters were lower after three cycles in the AI group; this difference persisted after six cycles as well $(p<0.05$, Figure 5). Six patients $(12,7 \%)$ needed at least one erythrocytes transfusion in the course of Ra-223 therapy; all of them belonged to the AI subgroup; moreover, all patients with grade III bone marrow toxicity and the one patient with grade IV leukopenia/neutropenia belonged to this subpopulation as well (see Figure 4 for details).

3.6. MANOVA. INV\% ratio predicted onset of grade III/IV hematologic toxicity at the whole-body $(p<0.05)$ and in the appendicular skeleton analysis $(p<0.01)$. Moreover, presence of AI predicted the onset of bone marrow toxicity $(p<0.005)$.

Similarly, mean $B_{\mathrm{Vol}}$ counts predicted grade III/IV hematologic toxicity in the whole-body as well as in the appendicular segments $(p<0.05)$ (see Table 3 for details). 

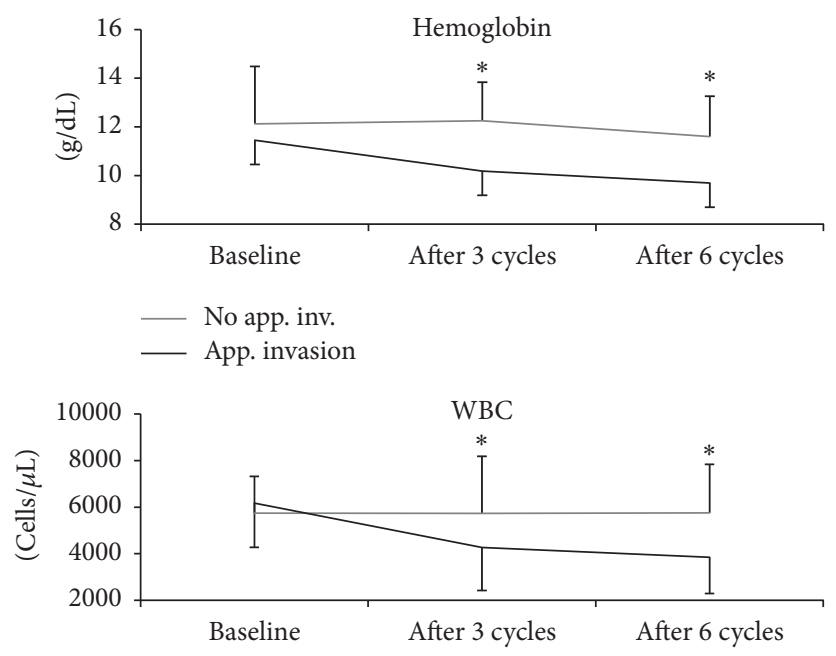

- No app. inv.

- App. invasion

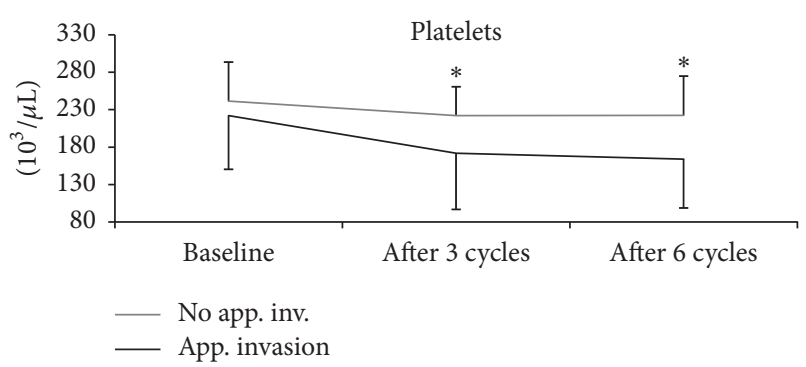

FIGURE 5: Course of blood values over time in patients with or without significant appendicular skeleton invasion. PLT $=$ platelets; WBC $=$ leukocytes. ${ }^{*} p<0.05$.

TABLE 3: Results of multivariate analysis for grade III/IV hematologic toxicity.

\begin{tabular}{|c|c|c|c|}
\hline Dependent variable & Degrees of freedom & $F$ value & $p$ value \\
\hline Age & 1 & 2,555 & NS \\
\hline Weight & 1 & 1,978 & NS \\
\hline PSA level & 1 & 3,637 & NS \\
\hline Superscan & 1 & 0,33 & NS \\
\hline$B_{\mathrm{Vol}}$ counts & 1 & 9,346 & 0,016 \\
\hline Axial $B_{\mathrm{Vol}}$ counts & 1 & 0,134 & NS \\
\hline Appendicular $B_{\mathrm{Vol}}$ counts & 1 & 5,318 & 0,045 \\
\hline$M_{\mathrm{Vol}}$ mean counts & 1 & 1,374 & NS \\
\hline Axial $M_{\mathrm{Vol}}$ mean counts & 1 & 0,708 & NS \\
\hline Appendicular $M_{\mathrm{Vol}}$ mean counts & 1 & 2,933 & NS \\
\hline INV\% & 1 & 9,881 & 0,014 \\
\hline INV\% (axial skeleton) & 1 & 1,509 & NS \\
\hline INV\% (appendicular skeleton) & 1 & 12,366 & 0,008 \\
\hline Appendicular invasion $(\mathrm{INV} \%>$ median $)$ & 1 & 16 & 0,004 \\
\hline
\end{tabular}

\section{Discussion}

The present paper introduces a computational method, based on segmentation analysis, to estimate the skeletal tumor burden and the dose to the hematopoietic bone marrow, situated in the trabecular bone niches. Our results confirm that both metastatic invasion and delivered activity to the trabecular bone can affect the bone marrow function.

In this study, we considered a patient population that presents a relevant metastatic invasion. On average, onethird of the available intraosseous space was occupied by tumor localizations. Previous studies have shown that normal 
hematopoiesis roughly requires one-third of the trabecular intraosseous space [17]. In the scenario of a $30 \%$ metastatic invasion, a hematopoietic volume expansion would still be possible.

However, patients presenting a skeletal metastasization (as calculated by the INV\% score) reaching the high end of the spectrum (i.e., 60-70\%) could not be able to react to a cytotoxic insult. Moreover, previous chemo- or radiotherapy could have already partly impaired the bone marrow reserve. As the mechanisms of metastasization generally affect the axial skeleton first [29], the presence of a massive metastatic component within the long bone could reflect a scenario of significant skeletal invasion [30].

In order to clarify the possible mechanisms of Ra-223 toxicity, a posttherapy bone marrow dosimetry would be necessary. However, the emission spectrum of this isotope does not allow for quantification in the clinical setting, as the injected activity is low and the fraction of emitted gamma-photons is too scarce. Given the reported similarity between Ra-223 and the $99 \mathrm{mTc}$-based bone-seeking tracer, we analyzed the pattern of the diagnostic tracer distribution in the cancellous bone of these subjects. The analysis showed that the trabecular bone activity could exhibit a 4-fold variation among subjects. Moreover, subjects showing a more intense uptake in the pretherapy bone SPECT/CT had a greater decline of hematologic parameters. This correlation was especially observed in the long bones, probably reflecting the above-described mechanisms, entailing a delocalization of active bone marrow from the axial skeleton to the long bones [25, 31-34], occurring in patients with considerable metastatic invasion.

As a matter of fact, the activity rate measured within the trabecular bones was strictly dependent on the entity of the metastatic invasion: the more the intraosseous spaces were occupied by tumor localizations, the higher the activity measured was in the apparently unaffected trabecular tissue. This could reflect either a tendency to instability in a skeleton whose static mechanisms are impaired by the osteoblastic reactions $[18,19,35]$ or, possibly, the presence of microscopic metastatic invasion that cannot yet be demonstrated at morphological imaging [36]. Independently of the pathophysiological considerations, these data suggest that a massive presence of metastases can impair bone marrow function during radionuclide therapy in two ways: by restricting the space available for bone marrow expansion and by indirectly increasing the dose to the unaffected trabecular bone.

The hereby-presented data introduce the concept of CTbased tumor burden analysis. This method was developed so as to reduce intercenters as well as interdevices variations. Nevertheless, the concept of a correlation between tumor burden and bone marrow failure can be applied to any tumor volume measurement method [15] and to any form of radioisotope therapy (such as Sm-153).

This study presents some limitations. It is a retrospective study, enrolling a relatively low number of patients. Therefore, incidence of bone marrow toxicity that was reported here cannot be directly related to those of larger trials $[10,11]$. However, previous trials considered a more diverse prostate cancer cohort, while the current study focused on the scenario of disseminated skeletal invasion. In this line, it confirms and extends on a computational perspective the results reported on planar scans in a similar cohort of patients [15].

Also, the representation of trabecular activity and blood parameter decline, as shown in Figure 3, is somewhat skewed; it must be accounted that the study analyzed a cohort of patients who had undergone different treatment scheme with a different duration before Ra-223 and whose baseline hematopoietic reserve could have been impaired by these therapies. However, the multivariate analysis confirms that trabecular tropism for bone-seeking tracers is an independent risk factor for bone marrow toxicity, at least in the setting of advanced disease.

Given the relatively low spatial resolution of SPECT, it cannot be excluded that radioactivity in osteoblastic tumor areas can at least partially contribute to the trabecular bone uptake, due to partial volume effect. This is unavoidable and represents a known limitation of the computational approach, especially when applied to SPECT/CT. However, the measured activity within metastases was not predictive of subsequent bone marrow failure, while the activity within trabecular bone is predictive. According to these considerations, the contribution of the radioactivity within the metastases is therefore less likely to have caused significant alterations on the counts within the trabecular bone. Moreover, as the segmentation method is based on CT images, it can be translated onto PET/CT data, in order to obtain a better spatial resolution.

\section{Conclusions}

Computational analysis of SPECT/CT images can estimate the degree of skeletal metastatic invasion, as well as the radioactivity concentration in the trabecular bone, where the bone marrow is located. The analysis of these parameters suggests that, in patients with a high skeletal tumor burden, the incidence of bone marrow failure in the course of radionuclide therapy could be higher than what reported previously. Further prospective studies are needed in order to confirm these data. The computational analysis could be used, in selected cases, to complement the visual evaluation and to possibly personalize the therapeutic protocols.

\section{Conflicts of Interest}

The authors declare that there are no conflicts of interest regarding the publication of this paper.

\section{References}

[1] P. H. Lange and R. L. Vessella, "Mechanisms, hypotheses and questions regarding prostate cancer micrometastases to bone," Cancer and Metastasis Reviews, vol. 17, no. 4, pp. 331-336, 1999.

[2] A. de la Taille, L. Martínez-Piñeiro, P. Cabri, A. Houchard, and J. Schalken, "Factors predicting progression to castrateresistant prostate cancer in patients with advanced prostate cancer receiving long-term androgen-deprivation therapy," $B J U$ International, vol. 119, no. 1, pp. 74-81, 2017. 
[3] J. S. de Bono, H. I. Scher, R. B. Montgomery et al., "Circulating tumor cells predict survival benefit from treatment in metastatic castration-resistant prostate cancer," Clinical Cancer Research, vol. 14, no. 19, pp. 6302-6309, 2008.

[4] W.-X. Qi, Z. Shen, and Y. Yao, "Docetaxel-based therapy with or without estramustine as first-line chemotherapy for castration-resistant prostate cancer: A meta-analysis of four randomized controlled trials," Journal of Cancer Research and Clinical Oncology, vol. 137, no. 12, pp. 1785-1790, 2011.

[5] K. McKeage, "Docetaxel: a review of its use for the firstline treatment of advanced castration-resistant prostate cancer," Drugs, vol. 72, no. 11, pp. 1559-1577, 2012.

[6] H. Jadvar, S. Challa, D. I. Quinn, and P. S. Conti, "One-year postapproval clinical experience with radium-223 dichloride in patients with metastatic castrate-resistant prostate cancer," Cancer Biotherapy and Radiopharmaceuticals, vol. 30, no. 5, pp. 195-199, 2015.

[7] G. Henriksen, K. Breistol, O. S. Bruland, O. Fodstad, and R. H. Larsen, "Significant antitumor effect from bone-seeking, alphaparticle-emitting (223) Ra demonstrated in an experimental skeletal metastases model," Cancer Research, vol. 62, no. 11, pp. 3120-3125, 2002.

[8] J. A. Carrasquillo, J. A. O’Donoghue, N. Pandit-Taskar et al., "Phase I pharmacokinetic and biodistribution study with escalating doses of ${ }^{223} \mathrm{Ra}$-dichloride in men with castrationresistant metastatic prostate cancer," European Journal of Nuclear Medicine and Molecular Imaging, vol. 40, no. 9, pp. 1384-1393, 2013.

[9] N. Pandit-Taskar, S. M. Larson, and J. A. Carrasquillo, "Boneseeking radiopharmaceuticals for treatment of osseous metastases, part 1: alpha therapy with 223Ra-dichloride," Journal of Nuclear Medicine, vol. 55, no. 2, pp. 268-274, 2014.

[10] P. Hoskin, O. Sartor, J. M. O’Sullivan et al., "Efficacy and safety of radium-223 dichloride in patients with castration-resistant prostate cancer and symptomatic bone metastases, with or without previous docetaxel use: A prespecified subgroup analysis from the randomised, double-blind, phase 3 ALSYMPCA trial," The Lancet Oncology, vol. 15, no. 12, pp. 1397-1406, 2014.

[11] O. Sartor, R. Coleman, S. Nilsson et al., "Effect of radium223 dichloride on symptomatic skeletal events in patients with castration-resistant prostate cancer and bone metastases: results from a phase 3, double-blind, randomised trial," The Lancet Onclogy, vol. 15, pp. 738-746, 2014.

[12] R. F. Hobbs, H. Song, C. J. Watchman et al., "A bone marrow toxicity model for 223Ra alpha-emitter radiopharmaceutical therapy," Physics in Medicine and Biology, vol. 57, no. 10, pp. 3207-3222, 2012.

[13] P. Thapa, D. Nikam, T. Das, G. Sonawane, J. P. Agarwal, and S. Basu, "Clinical efficacy and safety comparison of $177 \mathrm{Lu}-$ EDTMP with 153Sm-EDTMP on an equidose basis in patients with painful skeletal metastases," Journal of Nuclear Medicine, vol. 56, no. 10, pp. 1513-1519, 2015.

[14] M. Pacilio, G. Ventroni, C. Basile, P. Ialongo, D. Becci, and L. Mango, "Improving the dose-myelotoxicity correlation in radiometabolic therapy of bone metastases with $153 \mathrm{Sm}$ EDTMP," European Journal of Nuclear Medicine and Molecular Imaging, vol. 41, no. 2, pp. 238-252, 2014.

[15] M. Miederer, C. Thomas, J. Beck et al., "Haematopoietic toxicity of radium-223 in patients with high skeletal tumour burden," Nuklearmedizin/Nuclear Medicine, vol. 54, no. 5, pp. 197-203, 2015.
[16] J. N. Graff and T. M. Beer, "Pharmacotherapeutic management of metastatic, castration-resistant prostate cancer in the elderly: focus on non-chemotherapy agents," Drugs \& Aging, vol. 31, no. 12, pp. 873-882, 2014.

[17] G. Sambuceti, M. Brignone, C. Marini et al., "Estimating the whole bone-marrow asset in humans by a computational approach to integrated PET/CT imaging," European Journal of Nuclear Medicine and Molecular Imaging, vol. 39, no. 8, pp. 1326$1338,2012$.

[18] M. H. Weber, S. Burch, J. Buckley et al., "Instability and impending instability of the thoracolumbar spine in patients with spinal metastases: A systematic review," International Journal of Oncology, vol. 38, no. 1, pp. 5-12, 2011.

[19] M. J. Scheyerer, C. Pietsch, S. M. Zimmermann, G. Osterhoff, H.-P. Simmen, and C. M. L. Werner, "SPECT/CT for imaging of the spine and pelvis in clinical routine: A physician's perspective of the adoption of SPECT/CT in a clinical setting with a focus on trauma surgery," European Journal of Nuclear Medicine and Molecular Imaging, vol. 41, no. 1, pp. S59-S66, 2014.

[20] M. Pacilio, G. Ventroni, G. De Vincentis et al., "Dosimetry of bone metastases in targeted radionuclide therapy with alpha-emitting 223Ra-dichloride," European Journal of Nuclear Medicine and Molecular Imaging, vol. 43, no. 1, pp. 21-33, 2016.

[21] S. M. Larson, "EXINI quantitative bone scan index: Expanded utility for the planar radionuclide bone scan," Journal of Nuclear Medicine, vol. 57, no. 1, pp. 5-6, 2016.

[22] E. C. Etchebehere, J. C. Araujo, D. R. Milton et al., "Skeletal tumor burden on baseline 18F-fluoride PET/CT predicts bone marrow failure after 223Ra therapy," Clinical Nuclear Medicine, vol. 41, no. 4, pp. 268-273, 2016.

[23] K. Agrawal, F. Marafi, G. Gnanasegaran, H. Van Der Wall, and I. Fogelman, "Pitfalls and limitations of radionuclide planar and hybrid bone imaging," Seminars in Nuclear Medicine, vol. 45, no. 5, pp. 347-372, 2015.

[24] NCI N, DHHS. Common Terminology Criteria for Adverse Events v4.0 National Cancer Institute: NIH publication; 2009:\# 09-7473.

[25] F. Fiz, C. Marini, C. Campi et al., "Allogeneic cell transplant expands bone marrow distribution by colonizing previously abandoned areas: An FDG PET/CT analysis," Blood, vol. 125, no. 26, pp. 4095-4102, 2015.

[26] F. Fiz, C. Marini, R. Piva et al., "Adult advanced chronic lymphocytic leukemia: Computational analysis of whole-body CT documents a bone structure alteration," Radiology, vol. 271, no. 3, pp. 805-813, 2014.

[27] C. Marini, S. Bruno, F. Fiz et al., "Functional activation of osteoclast commitment in chronic lymphocytic leukaemia: a possible Role for RANK/RANKL pathway," Scientific Reports, vol. 7, no. 1, 2017.

[28] A. V. D’Amico, R. Whittington, S. Bruce Malkowicz et al., "Biochemical outcome after radical prostatectomy, external beam radiation therapy, or interstitial radiation therapy for clinically localized prostate cancer," Journal of the American Medical Association, vol. 280, no. 11, pp. 969-974, 1998.

[29] N. Wang, F. E. Docherty, H. K. Brown et al., "Prostate cancer cells preferentially home to osteoblast-rich areas in the early stages of bone metastasis: evidence from in vivo models," Journal of Bone and Mineral Research, vol. 29, no. 12, pp. 26882696, 2014.

[30] G. Knudson, G. Grinis, V. Lopez-Majano et al., "Bone scan as a stratification variable in advanced prostate cancer," Cancer, vol. 68, no. 2, pp. 316-320, 1991. 
[31] G. Hudson, "Effect of Hypoxia on Bone-Marrow Volume," British Journal of Haematology, vol. 4, no. 3, pp. 239-248, 1958.

[32] L. Ollivier, S. Gerber, D. Vanel, H. Brisse, and J. Leclère, "Improving the interpretation of bone marrow imaging in cancer patients," Cancer Imaging, vol. 6, no. 1, pp. 194-198, 2006.

[33] S. C. Kim, B. R. Krynyckyi, J. Machac, and C. K. Kim, "Patterns of red marrow in the adult femur," Clinical Nuclear Medicine, vol. 31, no. 12, pp. 739-741, 2006.

[34] F. M. Gonzalez, J. Mitchell, E. Monfred, T. Anguh, and M. Mulligan, "Knee MRI patterns of bone marrow reconversion and relationship to anemia," Acta Radiologica, vol. 57, no. 8, pp. 964-970, 2016.

[35] A. Sekita, A. Matsugaki, and T. Nakano, "Disruption of collagen/apatite alignment impairs bone mechanical function in osteoblastic metastasis induced by prostate cancer," Bone, vol. 97, pp. 83-93, 2017.

[36] X. Bi, J. A. Sterling, A. R. Merkel, D. S. Perrien, J. S. Nyman, and A. Mahadevan-Jansen, "Prostate cancer metastases alter bone mineral and matrix composition independent of effects on bone architecture in mice - A quantitative study using microCT and Raman spectroscopy," Bone, vol. 56, no. 2, pp. 454-460, 2013. 


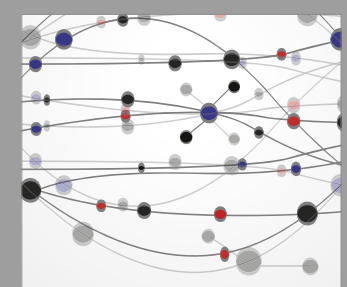

The Scientific World Journal
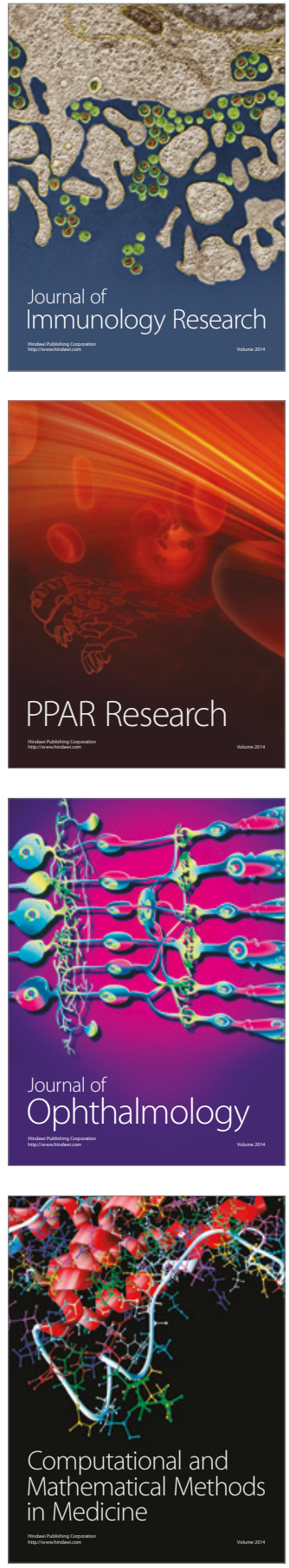

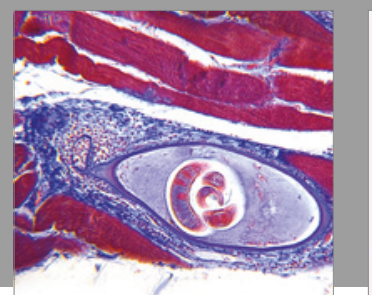

Gastroenterology Research and Practice
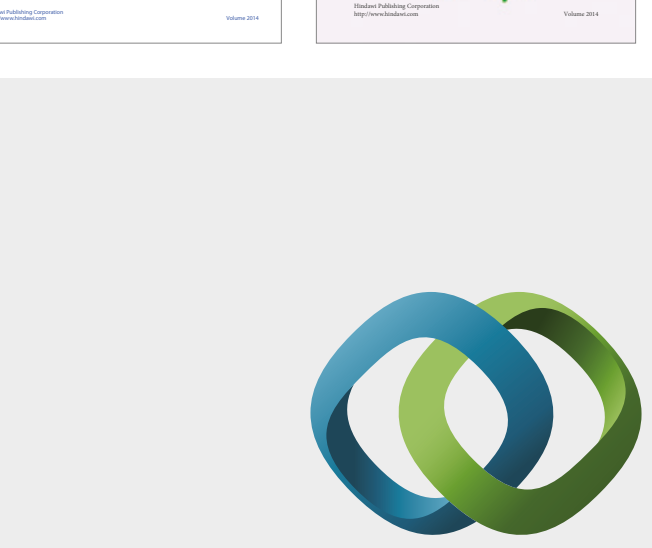

\section{Hindawi}

Submit your manuscripts at

https://www.hindawi.com
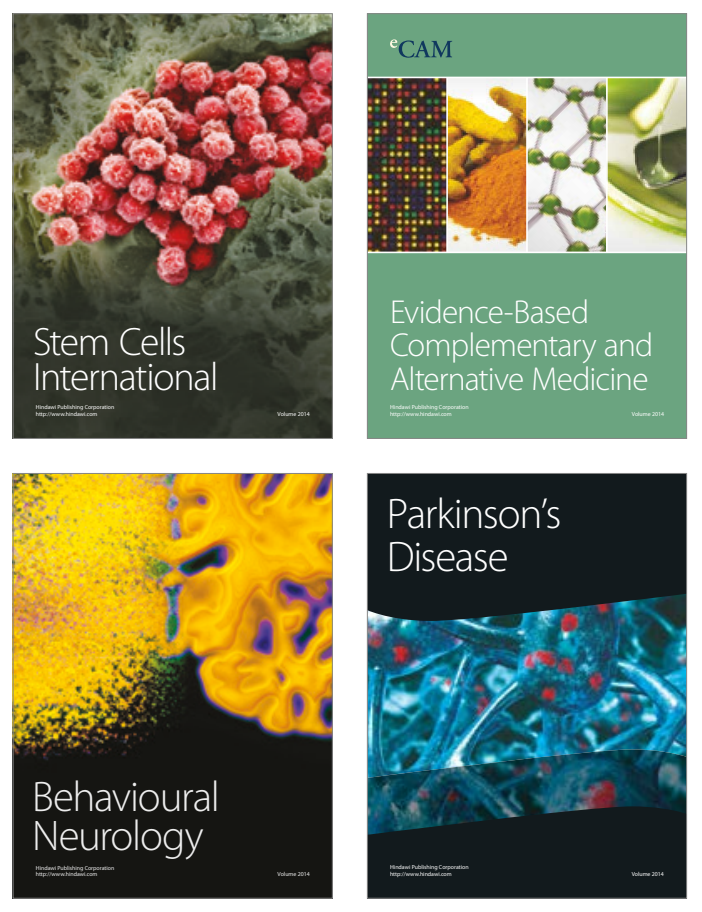
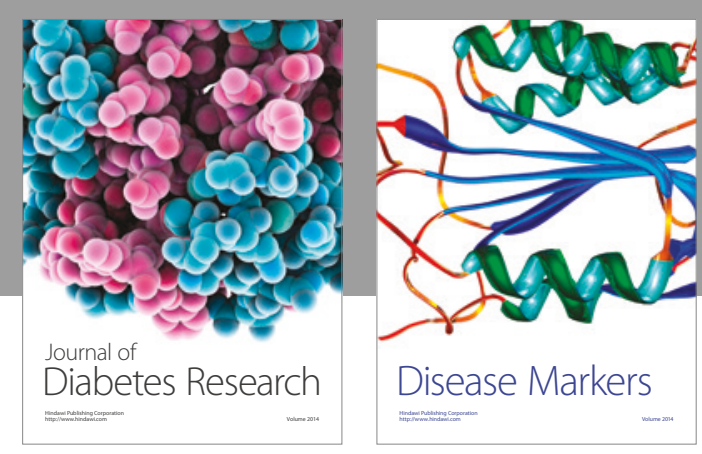

Disease Markers
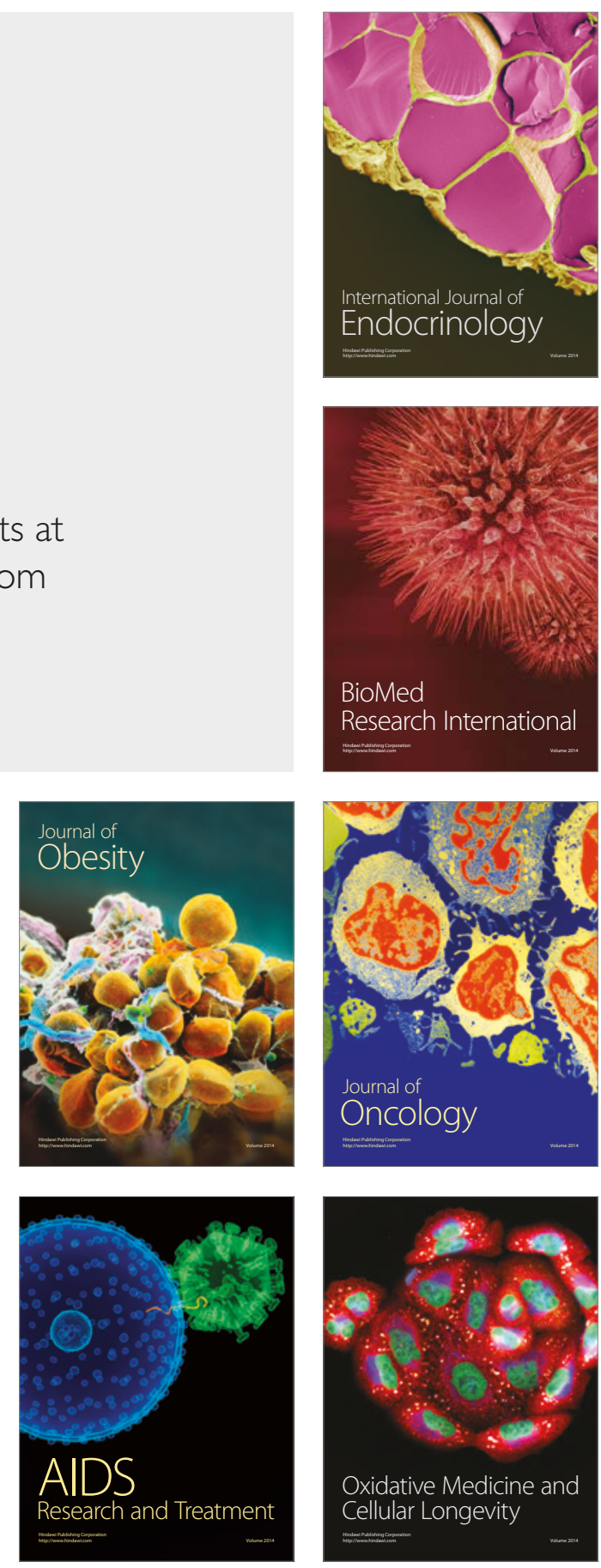